FM 11:

Global Coordination of Ground and Space Astrophysics and Heliophysics 


\title{
Global Coordination: What are the Next Steps?
}

\author{
David Spergel $^{1}$ and Robert Williams ${ }^{2}$ \\ ${ }^{1}$ Princeton University Observatory, Peyton Hall, Ivy Ln, Princeton NJ 08544-1001, New Jersey \\ (NJ) United States \\ email: dns@astro.princeton.edu \\ ${ }^{2}$ Space Telescope Science Institute, 3700 San Martin Drive,Baltimore MD \\ 21218-2410,Maryland (MD), United States \\ email: wms@stsci.edu
}

From the transit expeditions of 1761 to JWST, ALMA, and the SKA, international projects have played an important role in driving astronomy and heliophysics. Over the past two decades, the increasing complexity and cost of new facilities, the constrained amount of funding available from individual sources, and the rapidly increasing volume of data produced by newer facilities have made international collaboration on large groundand space-based facilities essential to moving the fields forward. As international cooperation becomes commonplace, data-sharing policies have become ever more important. All IAU members have a stake in the policy decisions made by nations and various scientific consortiums concerning data access and international collaborations. This focus meeting provided a forum to discuss how to improve coordination of global strategic planning in astronomy, astrophysics, and heliophysics in order to maximize the scientific return from research facilities.

How do we coordinate these international planning efforts? How do we balance national prioritizations with the increasing multi-national structures of our projects? How can and should we share the data produced both by these international collaborations and by other projects? How should we provide access to these facilities? Furthermore, the huge volume of data produced by current and future observation systems necessitates modes of research that have not heretofore figured prominently in astronomical and heliophysical research enterprises. The Advanced Technology Solar Telescope (ATST) will collect 3.65 petabytes in its first year of science operations while the Large Synoptic Survey Telescope (LSST) alone will produce $30 \mathrm{~TB}$ of data per night.

The potential benefit of enhanced international coordination is high. Much can be learned in astrophysics by adopting a broad-scoped approach, in which ground and spacebased facilities look at the same target with different wavelengths, timescales and technologies. Such an approach requires more resources than a single nation could maintain. Heliophysics has the added issue of coordinating truly global ground-based systems and space missions in various regions of the Sun-Earth system. In this context, Earth is an additional spacecraft embedded in its own space plasma environment. For the first time in history, we are capable of looking at a complicated coupled space system in its entirety, from the sun through the heliosphere, magnetosphere, ionosphere, and atmosphere down to the biosphere, in which we try to survive the present climate change. To study and understand the system around us is the ultimate benchmark to be able to understand other star-planet systems.

As the scope and scale of international collaboration on space science projects increases, challenges concerning managing data access and reciprocity between stakeholders have so far been dealt with on an ad hoc basis, project by project. The meeting consisted 
of a series of panels that bring together a diverse set of stakeholders representing the astronomical and heliophysics disciplines from around the world. Each of the sessions had significant audience participation and there were many fruitful exchanges between the panelists and between the panelists and the audience.

The FM11 focus meeting discussed the process that led successful international collaborations on projects such as the Cassini probe, ALMA, and Astro-H. Many national organizations and the European community have created processes of long-term prioritization, often based on synthesizing community views. The role of decadal reviews, now being undertaken by a number of countries, was emphasized, and agreed upon as the necessary basis for international coordination because of the national priorities that they establish. This said, Stuart Wyithe of Australia described the different philosophy that Australia's decade review undertook, in not prioritizing but rather setting out their strategy for achieving the goals of advancing astrophysics in the country.

Separate sessions were held on ground-based and space astronomy, with various speakers emphasizing the different situations that apply to ground vs. space telescopes, such as the crucial role of the space agencies in determining which space missions are approved. They concluded that global strategies for international coordination of ground projects should be handled separately and differently than global collaboration on space priorities and missions. An important aspect of international collaboration that was emphasized repeatedly by speakers from developing countries was that their participation as partners in 'big science' projects is the best way for small countries to enter the world of 'big time' astronomy. For developing countries international collaboration is essential for their meaningful introduction to design, fabrication, and operation of world class facilities.

Individual sessions were also devoted to the concepts of Open Skies and Open Data, with general agreement that science is advanced more when access to facilities is permitted on the basis of peer review without limitations based on other criteria such as cost compensation when necessary (largely for facilities constructed and operated from private funds). An obvious result from the discussions of all the above topics was that sharing of information in an open forum is very important for progress to be made on every aspect of global cooperation. The IAU should accept this as an important part of its mission in the future, in addition to being proactive in taking concrete steps to foster international collaboration.

The goal of the final session of FM11, devoted to 'Next Steps', was to conduct a panel discussion among five astronomers (N. Kaifu, R. Kraan-Korteweg, M. Colless, R. Bonnet, \& P. Ho) experienced in international collaborations and strategizing and have them give their assessment of ideas presented during the meeting. The panel was then charged with engaging the audience of interested participants in a discussion of specific actions that the IAU might take to facilitate cooperation and collaboration among nations, including the possible development of a common roadmap for future projects that would require international partners.

The panelists led off the discussion by proposing specific activities the IAU should undertake to enhance collaborations. These can be summarized as:

(a) sponsoring meetings, which could be full symposia or sessions of symposia devoted to research themes, where information on large projects being planned or under development is given,

(b) having the Division B Working Group on Future Large Scale Facilities taken on increased responsibility in helping develop international roadmaps in astronomy and infrastructure models for large collaborations, and to coordinate planning and information 
exchange between groups interested in committing to collaborations on large forefront facilities,

(c) creating a host website that serves as an information gathering and dissemination site for projects and groups seeking partnerships, and

(d) working with other organizations such as COSPAR to formulate an international roadmap, e.g., as has been done in Europe with Astronet, taking the priorities of the different national decadal reviews into account in a way that can serve as a focus for future mission and project planning.

The discussion between the panelists and the audience confirmed that IAU adoption of the above suggestions would provide significant support for projects of importance that, because of complexity and cost almost certainly require international collaboration, would move astronomy forward. It should be noted, however, that a few audience participants felt that the current system of establishing collaborations has worked well in the past and is not in need of improvement. The large majority of meeting attendees disagreed with this thinking, insisting that however successful astronomers may have been in forming collaborations in the past, the urgency of moving ahead with forefront facilities that are becoming prohibitively expensive requires serious efforts by the community.

An assessment by the FM11 SOC of the options available to the IAU to identify common priorities and facilitate international collaborations leads us to recommend that Division B and the Executive Committee agree to authorize the following course of actions:

- Given the importance of the WG on Future Large-Scale Facilities it may be appropriate to shift the WG from Div. B to the EC. This has been discussed with WG Chair Roger Davies and Div. B President Pietro Ubertini.

- The FL-SF WG is advised to create a mechanism, e.g., a website, that provides easy access to current information on large-scale facilities and includes announcements, offers of collaboration, etc., to be sent out to the community.

- Periodic symposia that are devoted to technological development and new facilities should be conducted by the IAU, much as SPIE holds meetings dedicated to instrumentation. The EC should also encourage sessions at selected symposia that are dedicated to future facilities.

- The IAU should consider a collaboration with COSPAR to define a process that could produce a roadmap identifying important scientific questions and mission concepts that would address them.

David Spergel Chair

Matthew Colless, Sarah Gibson, Lynne Hillenbrand,Cristina Mandrini, Hermann Opgenoorth, Xue Suijian, Yasushi Suto,Jean-Pierre Swings, Saku Tsuneta, Oskar von der Lühe, Patricia Whitelock,Robert Williams, Mei Zhang 